Delayed, distant skin lesions after transcranial direct current stimulation

\title{
Kortteenniemi, Aaron
}

2019

Kortteenniemi , A , Lehto , S M \& Javadi , A-H 2019 , ' Delayed, distant skin lesions after transcranial direct current stimulation ' , Brain Stimulation , vol. 12 , no. 1 , pp. 204-206 . https://doi.org/10.1016/j.brs

http://hdl.handle.net/10138/308859

https://doi.org/10.1016/j.brs.2018.10.018

publishedVersion

Downloaded from Helda, University of Helsinki institutional repository.

This is an electronic reprint of the original article.

This reprint may differ from the original in pagination and typographic detail.

Please cite the original version. 


\section{Delayed, distant skin lesions after transcranial direct current stimulation}

Keywords:

Transcranial direct current stimulation

Adverse effect

Safety

Skin lesion

Irritation contacted on day 18 and reported that the lesion was healing well. She was asked to send a photo, but she did not maintain communication after this point.

Participant \#2 - Similarly to Participant \#1, no signs of irritation were observable following tDCS. After two days, Participant \#2 developed a non-itchy, non-lumpy lesion (diameter ca. $6 \mathrm{~mm}$ ). In this case, the lesion was located on the palmar side of the wrist, while the electrode had been placed on the dorsal side. See Fig. 1c-d for images taken on days 2 and 22. The participant wished to take part in the second session, and the experimenter met with the participant for evaluation on day 48. In the safety evaluation, the participant still expressed a desire to participate in another session, and as the risk to the participant was estimated as low and no signs of skin irritation were observable, the participant was granted permission for a second session. The same stimulation protocol was applied. The participant was checked for adverse effects on days 50 and 55, i.e., days 2 and 7 following the second session. No skin reactions were observed.

Since 2013, we have used the same stimulation protocol over 400 times, and these two incidents of skin lesions have been the only observed cases. The participants had no history of such skin changes, and we thus considered these lesions to be stimulation induced. Previously, it has been suggested that the conditions of the skin-electrode impedance (effective contact size and impedance) are crucial in the formation of skin lesions [2]. While the safety features of the stimulator prevent the stimulation from continuing if the impedance rises too high, a decreased contact area and/or an insufficiently moistened electrode cannot be ruled out as causes for the first participant's lesions.

However, while the second participant's lesion resembled the other reported tDCS-induced lesions $[3,4]$ in timing and appearance, it did not form under the electrode. Although the lesion location was very unusual, we consider this lesion to have been caused by saline possibly having leaked out of the sponge, forming a conductive path through the bandage to the palmar aspect of the wrist. Another possibility for these lesions is a pre-existing skin defect not apparent on pre-stimulation examination. A small wound would provide a lowresistance path through the skin, which would concentrate the current in small area and could lead to injury [5].

Both delayed [6] and non-recurring [7] skin lesions have been documented in association with tDCS. Issues such as using tap water instead of saline, insufficiently moistened electrodes, electrode shape, non-uniform pressure and individual skin properties have been suggested as potential causes for these lesions, although the causes for delayed reactions remain open. Nevertheless, we are not aware of other reports of lesions on the opposite side of the limb to the electrode location. zinc oxide cream to promote healing. See Fig. $1 \mathrm{a}$ and $\mathrm{b}$ for images taken on days 2 and 6 . With the participant's permission, she was 
(a)

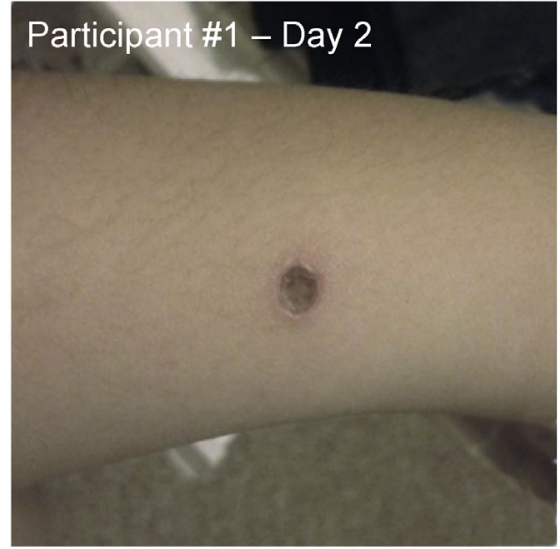

(c)

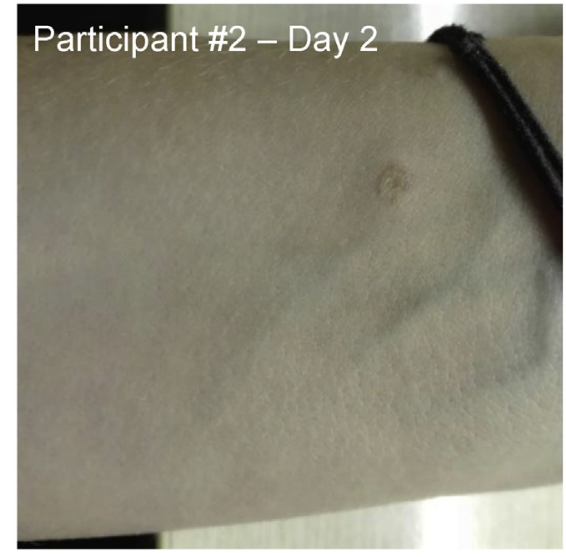

(b)

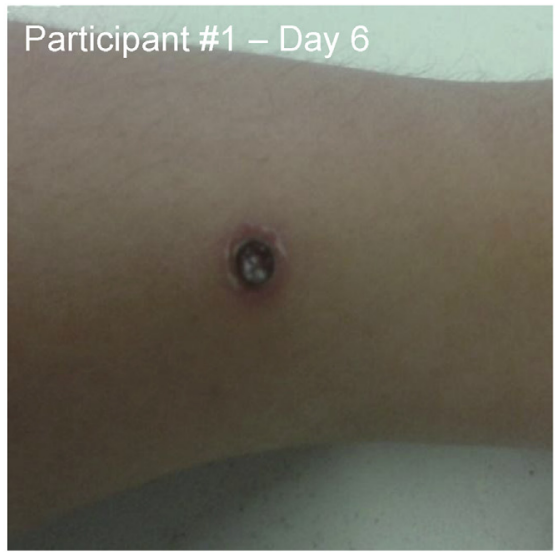

(d)

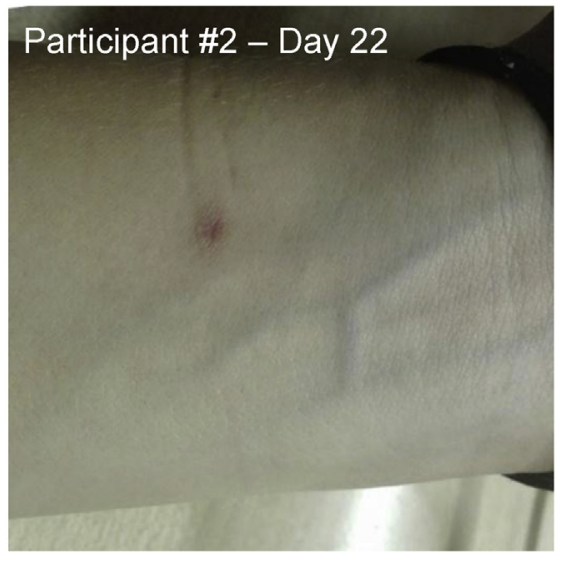

Fig. 1. Skin lesions of the two participants on different days following stimulation.

To conclude, these lesions, at least when adhering to safety guidelines, appear rare and manageable. Nonetheless, participants should be informed of the possibility of their occurrence. In addition, care should be taken when administering saline to the sponge electrodes. These two cases are may have followed the use of an improper amount of saline; the first participant may have received an insufficient amount, while the second participant's electrode may have been excessively moistened. Similarly, the condition of the electrodes should be monitored, as in our experience, the absorbance characteristics of the electrodes change with time, and harmful substances may accumulate [6]. To account for the possibility of pre-existing skin defects, the skin should be examined before stimulation using adequate care and lighting.

\section{Conflicts of interest}

We wish to confirm that there are no known conflicts of interest associated with this publication and there has been no significant financial support for this work that could have influenced its outcome. We confirm that the manuscript has been read and approved by all named authors and that there are no other persons who satisfied the criteria for authorship but are not listed. We further confirm that the order of authors listed in the manuscript has been approved by all of us.

\section{References}

[1] Woods AJ, Antal A, Bikson M, Boggio PS, Brunoni AR, Celnik P, et al. A technical guide to tDCS, and related non-invasive brain stimulation tools. Clin Neurophysiol 2015;127:1031-48. https://doi.org/10.1016/j.clinph.2015.11.012.
[2] Lagopoulos J, Degabriele R. Feeling the heat: the electrode-skin interface during DCS. Acta Neuropsychiatr 2008;20:98-100. https://doi.org/10.1111/j.16015215.2008.00274.x.

[3] Riedel P, Kabisch S, Ragert P, Von Kriegstein K. Contact dermatitis after transcranial direct current stimulation. Brain Stimul 2012;5:432-4. https://doi.org/ 10.1016/j.brs.2011.09.001.

[4] Palm U, Keeser D, Schiller C, Fintescu Z, Reisinger E, Padberg F, et al. Skin lesions after treatment with transcranial direct current stimulation (tDCS). Brain Stimul 2008;1:386-7. https://doi.org/10.1016/j.brs.2008.04.003.

[5] Aparício LVM, Guarienti F, Razza LB, Carvalho AF, Fregni F, Brunoni AR. A systematic review on the acceptability and tolerability of transcranial direct current stimulation treatment in neuropsychiatry trials. Brain Stimul 2016;9: 671-81. https://doi.org/10.1016/j.brs.2016.05.004.

[6] Frank E, Wilfurth S, Landgrebe M, Eichhammer P, Hajak G, Langguth B. Anodal skin lesions after treatment with transcranial direct current stimulation. Brain Stimul 2010;3:58-9. https://doi.org/10.1016/j.brs.2009.04.002.

[7] Rodríguez N, Opisso E, Pascual-Leone A, Soler MD. Skin lesions induced by transcranial direct current stimulation (tDCS). Brain Stimul 2014;7:765-7. https://doi.org/10.1016/j.brs.2014.06.005.

Aaron Kortteenniemi Institute of Clinical Medicine/Psychiatry, University of Eastern Finland, Kuopio, Finland

Soili M. Lehto

Institute of Clinical Medicine/Psychiatry, University of Eastern Finland, Kuopio, Finland

Department of Psychiatry, Kuopio University Hospital, Kuopio, Finland

Department of Psychology, Faculty of Medicine, University of Helsinki, Helsinki, Finland

Department of Psychiatry, Faculty of Medicine, University of Helsinki, Helsinki, Finland 
Amir-Homayoun Javadi* School of Psychology, University of Kent, Canterbury, United Kingdom

Institute of Behavioural Neuroscience, Department of Experimental Psychology, University College London, London, United Kingdom

School of Rehabilitation, Tehran University of Medical Sciences, Tehran, Iran
* Corresponding author. School of Psychology, Keynes College, University of Kent, Canterbury, CT2 7NP, United Kingdom. E-mail address: a.h.javadi@gmail.com (A.-H. Javadi).

16 October 2018

Available online 3 November 2018 